\title{
Modeling of the Earth atmosphere ionization by a galactic cosmic ray protons with RUSCOSMICS(C)
}

\author{
Maurchev E.A. \\ Polar Geophysical Institute \\ 26a, Academgorodok St., Apatity 184209, Apatity \\ E-mail: maurchev@pgia.ru \\ Balabin Yu.V. ${ }^{1}$ \\ Polar Geophysical Institute \\ 26a, Academgorodok St., Apatity 184209, Apatity \\ E-mail: balabin@pgia.ru
}

\begin{abstract}
One of main sources of the Earth's atmosphere ionization is the cosmic rays, galactic (GCR) and solar (SCR). Primary cosmic rays, consisting mainly of protons, ionize the upper layers of the atmosphere, and also interact with the nuclei of the surrounding matter (mainly nitrogen and oxygen), giving a secondary cosmic ray cascades, which in turn also participate in the ionization of the entire lower atmosphere. At the present day the cosmic ray (CR) physics uses a considerable variety of methods for the study of CR characteristics, and both a primary and a secondary fluxes, respectively. The experimental methods make the main contribution using various types of detectors, but the numerical methods complement it increasingly since the computer technology active development. This approach provides the researchers the most extensive information about the details of the process or phenomenon and allows you to make the most competent conclusions. This paper presents the use of the RUSCOSMIC@ software package based on the GEANT4 toolkit for calculation of the ionization rate for a various latitude and longitude values. The ionization profiles are plotted as a function of altitude and their specific numerical estimates are given.
\end{abstract}

35th International Cosmic Ray Conference - ICRC2017

10-20 July, 2017

Bexco, Busan, Korea

${ }^{1}$ Speaker 


\section{Introduction}

As a result of the interaction of the primary galactic cosmic rays (GCR) protons with the Earth's atmosphere substance (primarily nitrogen and oxygen), a secondary particle cascades are generated. From the altitudes of about $80 \mathrm{~km}$ to $\sim 15-20 \mathrm{~km}$ above sea level, the GCR protons lose their energy mainly through the ionization process. Starting with the atmosphere dense layers, protons with energies $\mathrm{E} \sim 1 \mathrm{GeV}$ are able to participate in inelastic collisions, transmitting up to half of their energy to secondary particles. This process can last from several to dozens of times, depending on the energy of the primary proton. Thus, using detectors of a charged component or a different configuration neutron monitors of , it is possible to fix secondary particles, partially or completely restoring information on primary CR protons [1, 2, $3,4]$. At the same time, it is quite difficult to calculate the parameters of secondary CR fluxes with the help of detectors, since this requires large statistics, which in turn leads to the need to conduct a number of expensive experiments. In addition to the high cost, it is not always possible to conduct an experiment in the right place and at the right time. Therefore, to study and evaluate the parameters of GCR in the Earth's atmosphere it is much more effective to use a combined technique that includes both real data and model experiments. This paper presents the results obtained in the calculation of the passage of proton GCR through the Earth atmosphere using the RUSCOSMICS $\odot$ software complex and also shows their comparison with the profiles obtained as a result of the balloons experiment [5].

\section{Methodology and results}

For the CR passage simulation through the atmosphere the RUSCOSMICS $\odot$ was used [6, 7]. A model particle source located at the upper boundary $(80 \mathrm{~km})$ of the Earth's atmosphere model was specified both a point source and a source with uniform spatial distribution in the xy projection. A typical visualization of the proton passage simulation through the Earth's atmosphere is shown in Fig. 1. It should be noted that cosmic rays include particles of different types, but most of them are protons, which are the main source of secondary cosmic ray generation. Therefore, in this work, a proton source model is used as the primary particles to reduce the load on the computer and increase the simulation speed. The energy characteristic (spectrum) of the primary GCR protons for the maximum and minimum of the 11-year solar activity cycle can be expressed in terms of the formula:

$$
F(x)=\frac{D \cdot E^{a}}{\left(10^{-2} \cdot E+B\right)}+C \cdot \exp \left(\frac{-E}{10}\right),\left(\mathrm{cm}^{2} \operatorname{sec~sr}^{2} \mathrm{MeV}\right)^{-1}
$$

Where $E$ is the kinetic energy of the proton, $D, B, C, \alpha$ are parameters that depend on the phase of the 11-year cycle. The differential spectra obtained by this formula are shown in the Fig.2. 


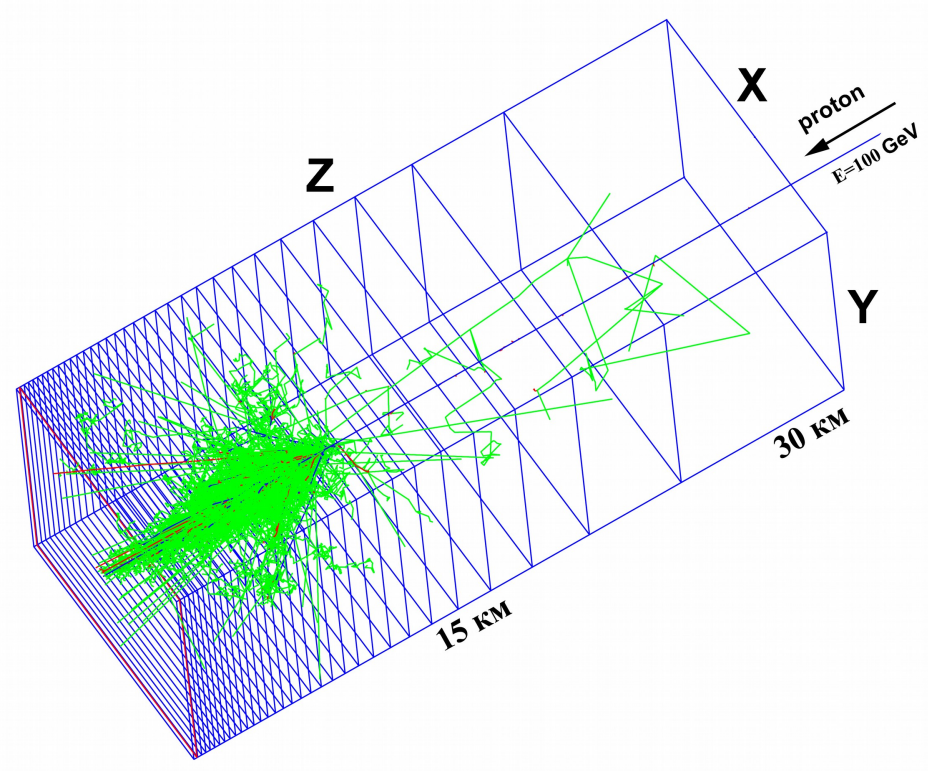

Figure 1. Visualization of the primary proton GCR interaction with energy $E=100 \mathrm{GeV}$ in the column of the matter of the Earth's atmosphere from $30 \mathrm{~km}$ to sea level (A) and the passage of a proton of the same energy through $80 \mathrm{~km}$ of the atmosphere (B), and the creation of a secondary particle cascades. The geometry in the form of layers is depicted in blue, the tracks of the particles are also divided in colors: a blue is positively charged, a red - negatively charged, a green - neutral.

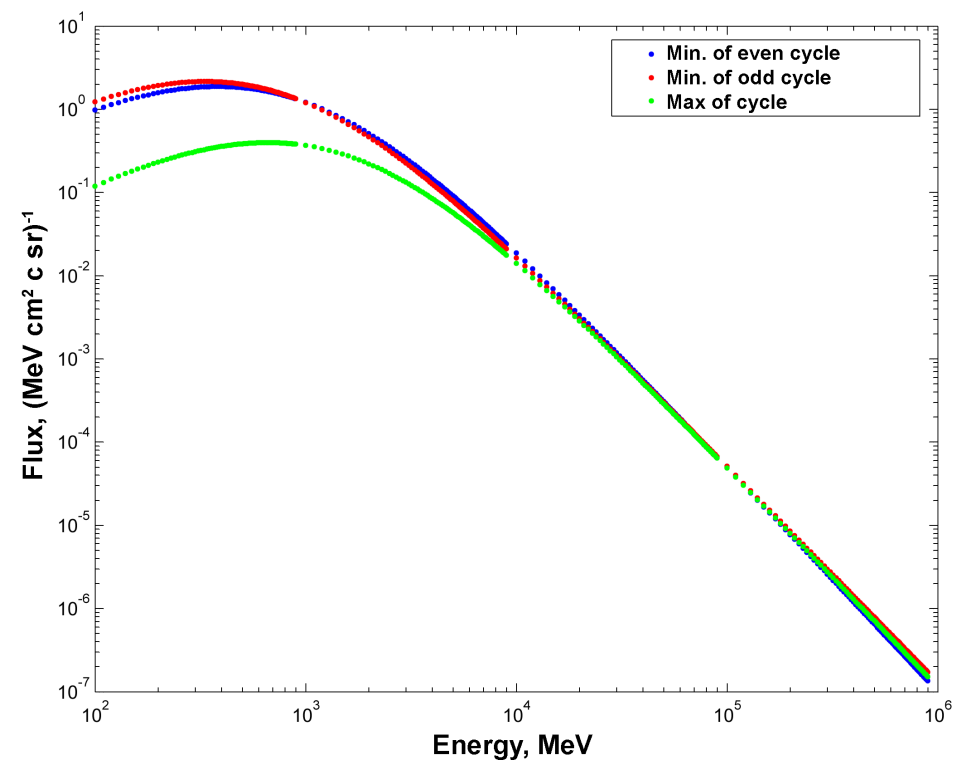

Figure 2. The differential energy spectra of GCR protons used to model cascades of secondary particles in the Earth's atmosphere: green dots for the maximum solar activity, blue and red for the minima of even and odd cycles, respectively. 
As a result of modeling the GCR protons passage through the Earth's atmosphere matter with the energies corresponding to the spectrum presented in Fig. 2 the secondary cosmic ray distributions (protons, neutrons, electrons, positrons, muons, photons) were obtained in the form of their intesity vs the atmosphere height and shown in the Fig. 3.

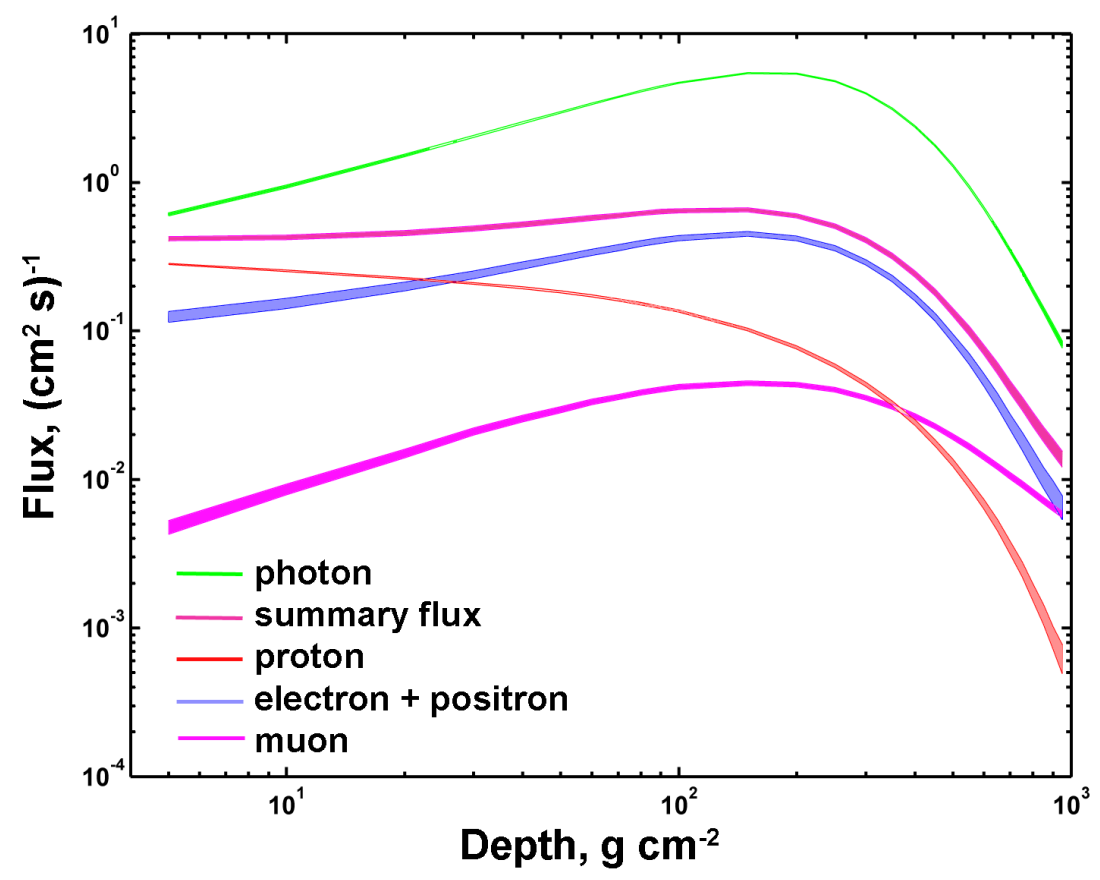

Figure 3. The altitude profiles for different types of particles, presented with an indication of the calculation error interval, obtained as a result of modeling the passage of GCR through the Earth's atmosphere. The line width means the total deviation from the mean and includes both the standard error and the internal error of the method.

For verification there is a huge database, representing the high-altitude profiles obtained as a result of the balloon experiments from the Earth level to the stratosphere [5]. One of the stations that regularly runs this equipment is located in the Apatity. Therefore, comparing these experimental data with simulation data, it is possible to verify the model as correct. The equipment placed on the balloon-probe is two Geiger-Muller counters working on coincidence, a barograph and a radio transmitter. Accordingly, the recorded profile will consist of the contribution of charged particles and $\sim 1 \%$ of gamma quanta, which is expressed in terms of the formula:

$$
J_{\text {summary }}=J_{p}+J_{\mp e}+J_{\mp \mu}+0.01 \cdot J_{\gamma}
$$

Where $J_{p}$ is the total proton flux, $J_{\mp e}$ is the total flux of electrons and positrons, $J_{\mp \mu}$ is the muon total flux, $J_{\gamma}$ is the total flux of gamma presented in the Fig. 3, taking into account the average statistical error in calculating are shown through the line width. The Fig. 4 compares the experimental data obtained by launching the balloon experiments on 04.09.2009 and 21.12.2009 in Apatity, Murmansk region, with the results of modeling the passage of GCR protons through the Earth's atmosphere with energy spectra corresponding to these periods. The ionization caused by the flows of these particles is presented in the Fig. $\mathbf{5}$ for different values of the geomagnetic cutoff rigidity. 

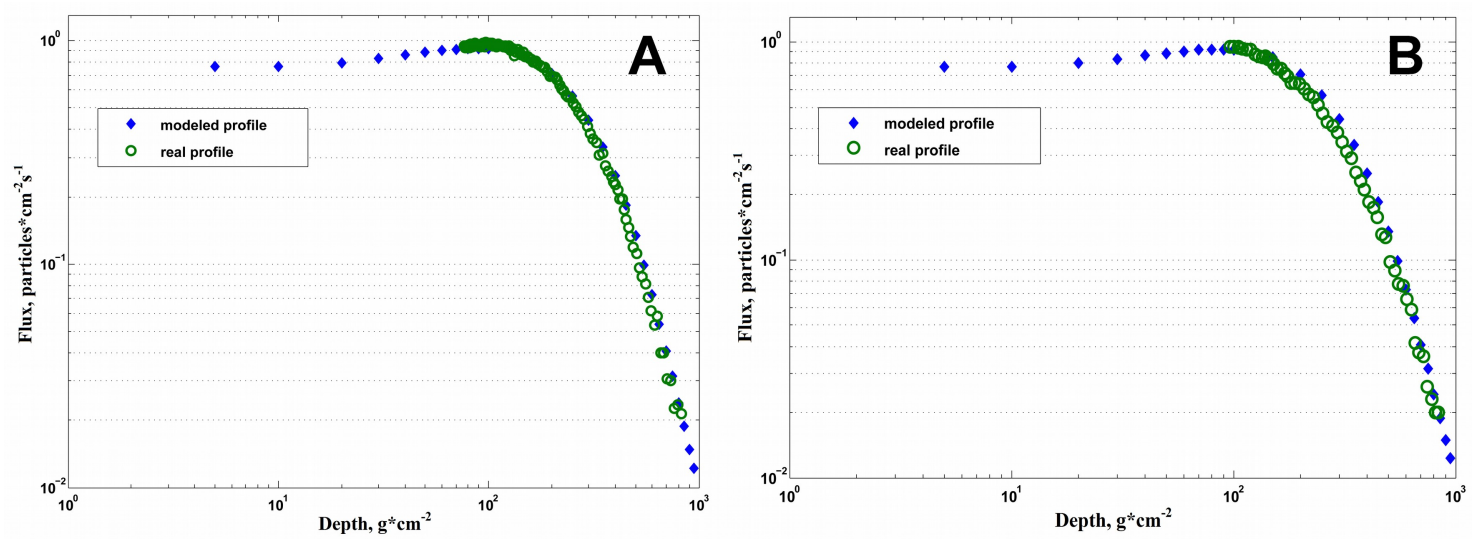

Figure 4. Comparison of experimental data (04.09.2009 (A) and 21.12.2009 (B)) and data obtained by modeling the passage of GCR particles through the Earth's atmosphere and processed in accordance with the formula 2.
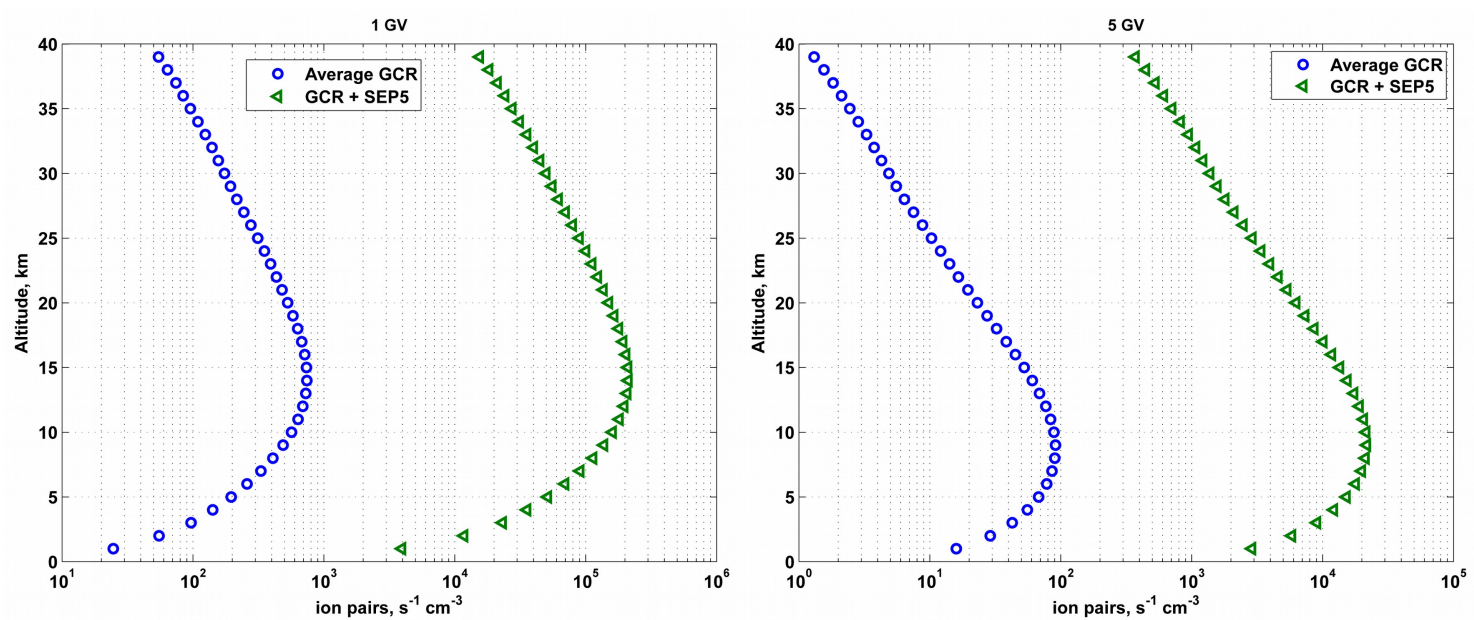

Figure 5. The ionization rate for various geomagnetic cutoff rigidity values, obtained as a result of modeling the passage of GCR particles through the Earth's atmosphere.

\section{Conclusion}

In this paper it was shown that the use of the RUSCOSMICS $(\subset)$ software complex is well suited not only for calculating the main radiation detectors, but also for calculating cosmic ray fluxes in the Earth's atmosphere, quantifying their intensity and calculating the ionization rate. Examples of such calculations were presented and compared with experimental data. In the future, the project is planned to be developed in the direction of increasing the speed of computation and universality.

\section{References}

[1] J. Perez-Peraza, A. Gallegos-Cruz, E. V. Vashenyuk, Yu. V. Balabin Relativistic proton production at the Sun in the October 28th, 2003 solar event, Adv. Space Res., 2005 
[2] J. Perez-Peraza, E. V. Vashenyuk, A. Gallegos-Cruz, Yu. V. Balabin, L. I. Miroshnichenko, Relativistic proton at the Sun in the 20 January 2005 solar event, Adv. Space Res., 41, 947-954, 2008

[3] B. Vashenyuk E. V., Yu. V. Balabin, L. I. Miroshnichenko, Relativistic solar protons in the ground level event of 23 February 1956: the new study, Adv.Space Res., 41, 926-935, 2008

[4] E.V. Vashenyuk, , Yu.V. Balabin, B.B. Gvozdevsky, Features of relativistic solar proton spectra derived from ground level enhancement events (GLE) modeling, Astrophysics and Space Sciences Transactions, Volume 7, Issue 4, 459-463, 2011

[5] V.S. Makhmutov, G.A. Bazilevskaya, Yu.I. Stozhkov, A.K. Svirzhevskaya, N.S. Svirzhevsky, Catalogue of electron precipitation events as observed in the long-duration cosmic ray balloon experiment, Journal of atmospheric and solar-terrestrial physics, 149, 258-276, 2016

[6] E.A. Maurchev, Yu.V. Balabin, B.B. Gvozdevsky, A new numerical model for investigating cosmic rays in the Earth's atmosphere, Bull. Russ. Acad. Sci. Phys., 2015

[7] E.A. Maurchev, Yu.V. Balabin RUSCOSMIC - the new software toolbox for detailed analysis of cosmic rays interactions with matter, Solar-Terrestrial Physics, 2, 4, 3-10, 2016 